\title{
Biomaterial-Facilitated Immunotherapy for Established Oral Cancers
}

David G. Leach ${ }^{1 \neq}$, Neeraja Dharmaraj ${ }^{2 \neq}$, Tania L. Lopez-Silva ${ }^{1}$, Jose Rodriguez Venzor ${ }^{2}$, Brett H. Pogostin, ${ }^{1}$ Andrew G. Sikora ${ }^{3}$, Jeffrey D. Hartgerink ${ }^{* 1}$, Simon Young*2

${ }^{1}$ Department of Chemistry, Department of Bioengineering, Rice University, Houston, TX 77005.

${ }^{2}$ Department of Oral \& Maxillofacial Surgery, The University of Texas Health Science Center at Houston School of Dentistry, TX 77054, USA; ${ }^{3}$ Department of Head and Neck Surgery, Division of Surgery, The University of Texas MD Anderson Cancer Center, Houston, TX 77030.

${ }^{\ddagger}$ Authors contributed equally

${ }^{*}$ Corresponding authors

Corresponding author: Simon Young (simon.young@uth.tmc.edu)

The University of Texas Health Science Center at Houston, School of Dentistry

Department of Oral \& Maxillofacial Surgery

7500 Cambridge St. Suite 6510, Houston, TX, 77054, USA

7134862568 tel

7134864333 fax

SUPPORTING INFORMATION 


\section{MATERIALS AND METHODS}

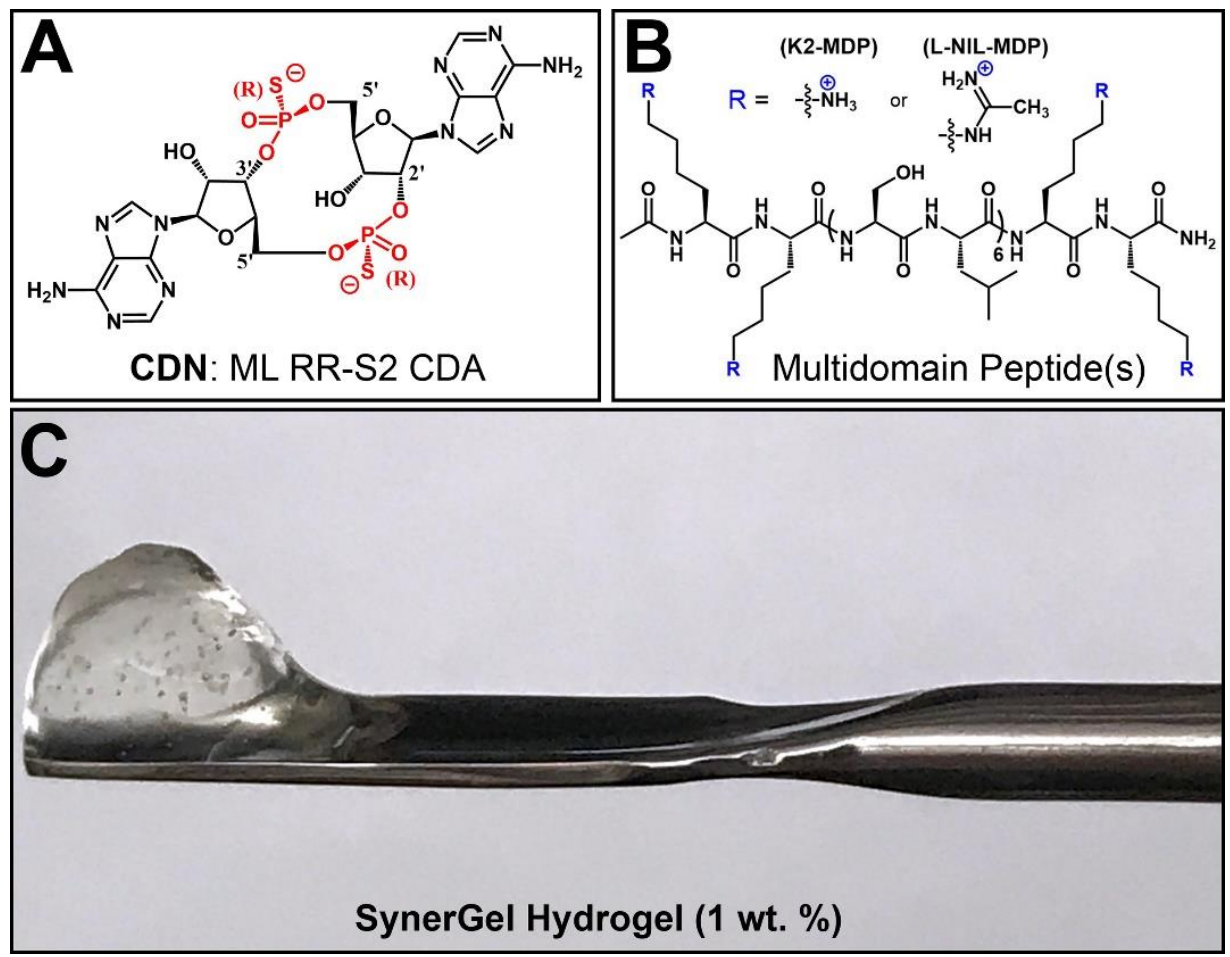

Figure S1. Key components of STINGel (K2-MDP+CDN) and SynerGel (L-NIL-MDP+CDN) formulations. (A) Chemical structure of small molecule STING agonist cyclic dinucleotide (CDN) ML RR-S2 CDA (highlighting in red the negatively charged thiophosphate linkages of the molecule). (B) Chemical structure of multidomain peptide backbone with R groups showing amino acid side chain chemistry of K2-MDP, bearing positively charged lysine amines, and L-NIL-MDP, bearing positively charged acetamidine groups that mimic the structure of the small molecule iNOS inhibitor L-NIL. (C) Image of the self-supporting, syringe injectable SynerGel hydrogel formed at 1 wt. $\%(10 \mathrm{mg} / \mathrm{mL})$ in buffered solution.

\section{Peptide synthesis}

All MDPs used in this study were synthesized by solid phase peptide synthesis with amidated C-termini and acetylated N-termini. The L-NIL-MDP was synthesized by on-resin side chain deprotection of acid-labile monomethoxytrityl ( $\mathrm{Mmt}$ ) protecting groups, followed by conversion of free lysine side chains to the L-NIL acetamidine functional group by reaction with excess ethyl acetimidate-HCl and DIEA as described previously. ${ }^{1}$ However, in previously reported syntheses, deprotection of the Mmt groups was performed using 10\% acetic acid in TFE/DCM, but in this study, use of 1\% TFA in DCM provided superior deprotection speed and higher percent conversion and was used throughout. For confirmation of successful synthesis, all peptides were analyzed by matrix-assisted laser desorption/ionization time of flight mass spectrometry (MALDI-TOF MS) using an Autoflex Bruker instrument (Supplementary Figure S2). For more detailed peptide synthesis methods of the multidomain peptides (MDPs) $\mathrm{K}_{2}(\mathrm{SL})_{6} \mathrm{~K}_{2}, \mathrm{O}_{5}(\mathrm{SL})_{6} \mathrm{O}_{5}, \mathrm{E}_{2}(\mathrm{SL})_{6} \mathrm{E}_{2}$, and the L-NIL-MDP, please refer to our previous publications. ${ }^{1-3}$ 

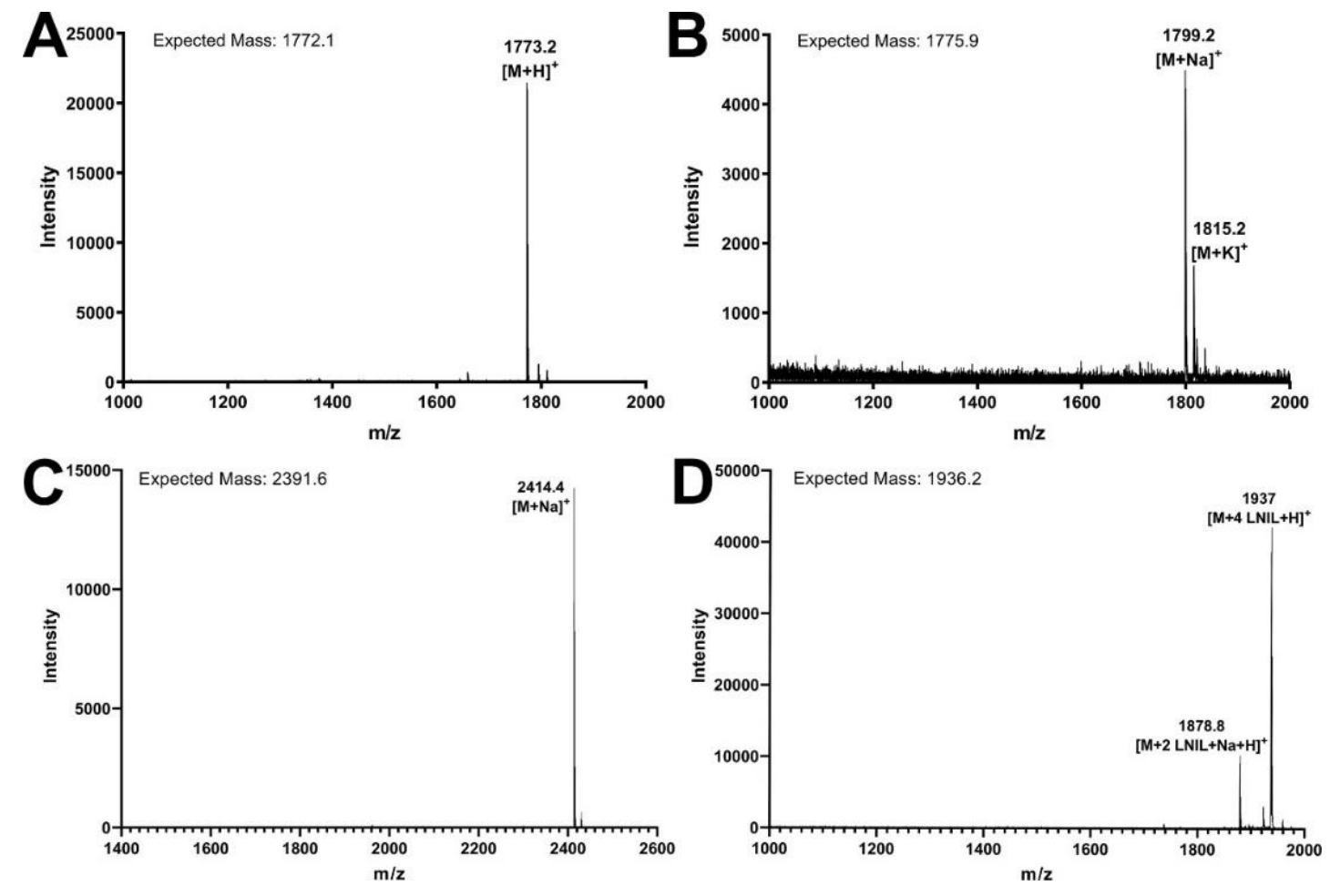

Figure S2. MALDI-TOF mass spectroscopy spectra for synthesized MDPs (A) $\mathrm{K}_{2}(\mathrm{SL})_{6} \mathrm{~K}_{2}$, (B) $\mathrm{E}_{2}(\mathrm{SL})_{6} \mathrm{E}_{2}$, (C) $\mathrm{O}_{5}(\mathrm{SL})_{6} \mathrm{O}_{5}$, (D) L-NIL-MDP $\left[\mathrm{K}^{\mathrm{LNIL}}{ }_{2}(\mathrm{SL})_{6} \mathrm{~K}^{\mathrm{LNIL}}{ }_{2}\right]$.

\title{
Hydrogel preparation and loading
}

\begin{abstract}
All chemicals not otherwise specified were purchased from Sigma-Aldrich (Sigma-Aldrich, St. Louis, MO). All sterile K2-MDP, L-NIL-MDP, E2MDP, and O5-MDP hydrogels were prepared first at $2 \mathrm{wt} . \%(20 \mathrm{mg} / \mathrm{mL})$ stock peptide solutions by adding sterile $298 \mathrm{mM}$ sucrose to lyophilized peptide mass, using vortexing and sonication to aid dissolution. Full dissolution of peptide mass usually occurred over 1-3 hours. Stock cyclic dinucleotide ML RR-S2 CDA (CDN) (MedChem Express, Monmouth Junction, NJ) was initially prepared at $2.67 \mu \mathrm{g} / \mu \mathrm{L}$ in endotoxin-free $\mathrm{H}_{2} \mathrm{O}$ or sterile 1X HBSS ( $3.64 \mathrm{mM}$, 4-fold higher than the final dose of $0.91 \mathrm{mM}, 0.67 \mathrm{ug} / \mathrm{uL}$, with a target dosage of $20 \mu \mathrm{g}$ CDN in $30 \mu \mathrm{L}$ of hydrogel). Concentration of CDN stocks was confirmed by UV-Vis. Formulations of collagen hydrogel were prepared according to the provided kit protocol (ECM675, EMD Millipore, Temecula, CA). Hyaluronic acid gel formulations were prepared according to the provide kit protocol (HyStem Scaffold Kit, HYSC010, Sigma-Aldrich). Sodium alginate (ICN218295, MP Biomedicals, Irvine, CA) hydrogels were prepared by crosslinking 1 wt.\% aqueous alginate with $0.1 \mathrm{M} \mathrm{CaCl}_{2}$. Matrigel formulations were made using the provided protocol (Corning Matrigel, 47743-715, VWR, Radnor, PA). Table S1 contains additional information in summary form.
\end{abstract}

Sterile 1X HBSS (Fisher Scientific, Hampton, NH) was used to form hydrogels for cationic MDPs (K2-MDP, L-NIL-MDP), and another solution of $45 \mathrm{mM} \mathrm{MgCl}_{2}$ in $1 \mathrm{X}$ HBSS was used with the anionic E2-MDP. To form all CDN drug-loaded hydrogels, 2 wt. \% peptide solutions were diluted 1:1 with $1 \mathrm{X}$ HBSS $+2 \mathrm{X} \mathrm{CDN}(1.82 \mathrm{mM} \mathrm{CDN})$, or $1 \mathrm{X} \mathrm{HBSS}+45 \mathrm{mM} \mathrm{Mg}{ }^{2+}+2 \mathrm{X}$ CDN based on the cationic or anionic nature of the peptide requirements for gelation. Final formulations contained $1 \mathrm{wt}$. \% peptide $(10 \mathrm{mg} / \mathrm{mL}$, approximately $5-6 \mathrm{mM}), 0.5 \mathrm{X} \mathrm{HBSS}, 1 \mathrm{X}$ CDN (0.91 mM) and $149 \mathrm{mM}$ sucrose. The neutral O5-MDP hydrogel was diluted to $1 \mathrm{wt} . \%$ with $1 \mathrm{X}$ HBSS + 2X CDN and hydrogelation was triggered by ultrasonication as described previously. ${ }^{2}$ Prepared gel formulations for in vivo experiments were slowly loaded using Monoject $300 \mu \mathrm{L}$ Insulin syringes (Covidien, Mansfield, MA) to avoid bubble formation (centrifuging down bubbles formed in hydrogels as necessary), and loaded syringes were allowed to equilibrate for $\sim 30$ min before injection. 


\section{Drug release kinetics}

For release kinetics studies of CDN release from various hydrogel formulations, aliquots of CDN-loaded gel ( $30 \mu \mathrm{L}$ each) were deposited into 96 well Falcon' flat bottom plates (Becton Dickinson Labware, Franklin Lakes, NJ). $30 \mu \mathrm{L}$ gel was pipetted to generate a cylindrical puck in each well for each experiment. The pipetted gels were allowed to shear recover for approximately 5-10 min before adding $200 \mu \mathrm{L}$ of the relevant buffer to the top of the gels (1X HBSS for all MDPs, 1X DPBS for collagen, Matrigel, $1 \mathrm{X} \mathrm{HBSS}$ with $0.1 \mathrm{M} \mathrm{CaCl}_{2}$ for alginate, etc. according to the gelation requirements of the material). The addition of buffer was defined as $t=0$ for the beginning of release kinetics experiments. Table S1 summarizes the material conditions and preparation methods used for various comparative CDN delivery kinetics studies. The final column of Table S1 on syringe injectability of the formed gels refers to if the hydrogel systems can be successfully injected through a small gauge needle in vivo and still form a durable hydrogel implant post injection. The viscoelastic MDP hydrogels are pre-gelled by mixing with multivalent salts (counterions), liquify upon shearing through needles, and shear recover back into hydrogels post injection in vivo. The collagen and Matrigel systems are kept as chilled liquids in the needle, syringe injected as a liquid, and form hydrogels by heat crosslinking in vivo due to body heat. The alginate and hyaluronic acid systems have to be chemically crosslinked by calcium or covalent thiol crosslinking, and cannot be syringe injected due to material thickness and strength (and lack of shear recovery). Such systems as tested in this report would likely have to be surgically implanted in vivo rather than injected, as has been done in multiple prior reports as referenced in the manuscript introduction.

Table S1. Summary of tested hydrogel systems for drug release kinetics studies.

\begin{tabular}{|c|c|c|c|c|}
\hline Sample & $\begin{array}{c}\text { Material } \\
\text { Concentration }\end{array}$ & $\begin{array}{c}\text { CDN } \\
\text { Concentration }\end{array}$ & Gelation Method & $\begin{array}{c}\text { Syringe } \\
\text { Injectable Gel }\end{array}$ \\
\hline Alginate & $10 \mathrm{mg} / \mathrm{mL}$ & $1 \mathrm{X}(0.91 \mathrm{mM})$ & $\begin{array}{l}\text { in situ } \mathrm{Ca}^{2+} \text { ionic } \\
\text { crosslinking }\end{array}$ & No \\
\hline HyAcid & $8 \mathrm{mg} / \mathrm{mL}$ & $0.8 X$ & $\begin{array}{c}\text { Thiol + PEG-DA } \\
\text { covalent crosslinking }\end{array}$ & No \\
\hline Collagen & $4 \mathrm{mg} / \mathrm{mL}$ & $1 X$ & $\begin{array}{l}\text { Heat induced } \\
\text { crosslinking }\end{array}$ & Gels in situ \\
\hline Matrigel & $10.8 \mathrm{mg} / \mathrm{mL}$ & $1 X$ & $\begin{array}{l}\text { Heat induced } \\
\text { crosslinking }\end{array}$ & Gels in situ \\
\hline K2 MDP & $10 \mathrm{mg} / \mathrm{mL}$ & $1 X$ & $\begin{array}{c}\mathrm{PO}^{3-} \text { ionic charge } \\
\text { shielding/crosslinking }\end{array}$ & Yes \\
\hline L-NIL MDP & $10 \mathrm{mg} / \mathrm{mL}$ & $1 X$ & $\begin{array}{c}\mathrm{PO}^{3-} \text { ionic charge } \\
\text { shielding/crosslinking }\end{array}$ & Yes \\
\hline E2 MDP & 10 mg/mL & $1 \mathrm{X}$ & $\begin{array}{c}\mathrm{Mg}^{2+} \text { ionic charge } \\
\text { shielding/crosslinking }\end{array}$ & Yes \\
\hline
\end{tabular}

A Nanodrop 2000C Spectrophotometer (Thermo Scientific) was used to measure drug release by UV absorbance at $259 \mathrm{~nm}$ for CDN ML RR-S2 $\mathrm{CDA}$, using an extinction coefficient of $24,000 \mathrm{M}^{-1} \mathrm{~cm}^{-1}(259 \mathrm{~nm})$. Absorbance measurements were obtained by removing $1 \mu \mathrm{L}$ from the top of the liquid buffers above the loaded gels, over time measuring the increase in absorbance. Over the course of the first 24 hours, release of CDN was measured usually every 1-2 hours and converted to total percent released, with additional measurements also made every 24 hours for up to a week to establish when equilibrium had been reached.

\section{Oscillatory rheology}

The mechanical properties of the studied peptide hydrogels were analyzed by oscillatory rheology, using a TA Instruments AR-G2 rheometer (TA Instruments, New Castle, DE). MDP hydrogel samples $150 \mu \mathrm{L}$ in volume (at $1 \mathrm{wt}$ \%) were prepared 1 day before rheological testing and allowed to equilibrate in a cut syringe. Hydrogels were then transferred from the syringe to the rheometer stage, and the rheometer was equipped with a $12 \mathrm{~mm}$ stainless-steel parallel plate with $1000 \mu \mathrm{m}$ gap height. As published previously, the following program was used to analyze the storage modulus $\left(\mathrm{G}^{\prime}\right)$ and loss modulus $\left(\mathrm{G}^{\prime \prime}\right)$ under various instrument conditions. ${ }^{4}$ Analysis of strain sweep used $0.01-200 \%$ applied strain at $1 \mathrm{rad} / \mathrm{s}$ frequency. Analysis of frequency sweep used $1 \%$ strain at $0.1-100 \mathrm{rad} / \mathrm{s}$. Analysis of shear recovery was performed by subjecting the hydrogel to $1 \%$ strain for $20 \mathrm{~min}$, then 
$200 \%$ for $1 \mathrm{~min}$, and lastly $1 \%$ for $20 \mathrm{~min}$. This process allowed for shear disruption of the hydrogel sample and timed monitoring of $\mathrm{G}^{\prime}$ and $\mathrm{G}^{\prime \prime}$ recovery post shear.

\section{Cell culture}

The murine oral cancer cell line, MOC1 was provided by Dr. Ravindra Uppaluri (Dana-Farber Cancer Institute, Harvard University, Boston, MA) and maintained as previously described. ${ }^{5}$ For in vitro experiments, mouse $\mathrm{MOC} 1$ cells were cultured at $37^{\circ} \mathrm{C}$ with $5 \% \mathrm{CO}_{2}$ in media routinely used for maintaining this cell line. ${ }^{5}$ For live-dead viability studies done using 2-D culture, peptide hydrogel $70 \mu \mathrm{L}$ in volume (thickness approximately 1.75 $\mathrm{mm}$ ) were pipetted into the wells of Lab-Tek 16 well chamber slides (Thermo Fisher, Rochester, NY). The gels were pipetted carefully into the bottom of the wells, and the plates were gently tapped down onto the bench top before shear recovery to flatten the pipetted gels and facilitate uniform spreading of the material. After pipetting, time was allowed for the samples to shear recover (5-10 min) before the addition of $200 \mu \mathrm{L}$ cell media and 5,000 MOC1 cells. Each time point (days 1, 3, 7) had separate slides prepared with duplicate gels. Cell media was replaced every 2 days, carefully pipetting to avoid dislodging the gel material. Live-dead analysis was performed using the procedure previously described, ${ }^{1}$ using $2 \mu \mathrm{M}$ Calcein AM (Thermo Fisher), $4 \mu \mathrm{M}$ Ethidium homodimer (Thermo Fisher), and $5 \mu \mathrm{g} / \mathrm{mL}$ Hoechst 33342 (BD Biosciences, San Jose, CA) in DPBS. Z-stack imaging with a Nikon A1 Confocal Microscope equipped with 40X water objective was used to image gels. Image processing was done with NIS Elements (Nikon Instruments, Melville, NY), and Imaris Cell Counting software (Bitplane, Concord, MA) was used to quantify live-dead percentages.

\section{Subcutaneous experiments and histology}

Female mice (C57BL/6J), purchased at age 8-12 weeks from The Jackson Laboratory, were used for subcutaneous experiments. HHousing for all mice was specific pathogen-free conditions, with standard temperature and lighting conditions and free access to provided food and water. All animal experiments were conducted according to NIH guidelines and with approval from Rice University's Institutional Animal Care and Use Committee (IACUC). Mice were anesthetized using standard practice isoflurane ( $2 \%$ carried by oxygen) and maintained on a nose cone with $2 \%$ isoflurane. Subcutaneous experiments were performed by injecting mice with $100 \mu \mathrm{L}$ MDP hydrogels in each of four localized sites in the dorsal flank subcutaneous space after hair was removed from the skin by shaving and the area was presterilized with swabs of $70 \%$ isopropyl alcohol. The mice were humanely euthanized by $\mathrm{CO}_{2}$ asphyxiation while anesthetized at days 3 and 7 , and the dorsal skin directly surrounding the hydrogel implant was removed. The skin and implant samples were then fixed overnight in $10 \%$ neutral buffered formalin, before being processed and paraffin embedded. Paraffin blocks were sectioned at $5 \mu \mathrm{m}$ thickness using a microtome, and staining was performed using standard Masson's trichrome kits.

\section{MOC orthotopic murine model of oral cancer}

MOC1 tumors were established by injecting $2 \times 10^{6}$ cells (70\%:30\% cells: Matrigel ${ }^{\circledR}$ Matrix) into the maxillary vestibule of the left oral cavity in $\mathrm{C} 57 \mathrm{BL} / 6 \mathrm{~J}$ mice. Once MOC1 tumors were established ( $4-5 \mathrm{~mm}$ diameter, typically requiring 5 days of growth), $30 \mu \mathrm{l}$ of SynerGel or controls were injected into the oral tumor site. Tumor growth was monitored by tumor size measurements (in diameter) taken at least two times per week using digital calipers to measure the longest dimension (single dimension). Data was then analyzed for Kaplan-Meier survival and tumor growth curves. All protocols were in accordance with the guidelines for humane treatment of laboratory animals by the National Institutes of Health, the Animal Welfare Committee and the Center for Laboratory Animal Medicine and Care (CLAMC) at the University of Texas Health Science Center at Houston. Excessive tumor burden, defined as tumor diameter reaching $12 \mathrm{~mm}$, tumor ulceration, or a weight loss of greater than $20 \%$ were factors that were considered for all endpoints in tumor growth and survival data.

\section{Statistical methods}

Statistical analyses were performed using Prism 7.0 (GraphPad, San Diego, CA). Statistical analyses for Kaplan-Meier survival curves were performed using the Log-rank/Mantel-Cox test. Statistical significance for tumor growth curves was determined by a two-way ANOVA test followed by selected comparison using Tukey's multiple comparison tests with multiple comparison correction. All $\mathrm{p}$ values were two-sided and $\mathrm{p}$ values less than 0.05 were considered significant. 


\section{References}

1. Leach, D. G.; Newton, J. M.; Florez, M. A.; Lopez-Silva, T. L.; Jones, A. A.; Young, S.; Sikora, A. G.; Hartgerink, J. D., Drug-Mimicking Nanofibrous Peptide Hydrogel for Inhibition of Inducible Nitric Oxide Synthase. ACS Biomater. Sci. Eng. 2019, 5 (12), 6755-6765. DOI: 10.1021/acsbiomaterials.9b01447.

2. $\quad$ Lopez-Silva, T. L.; Leach, D. G.; Li, I. C.; Wang, X.; Hartgerink, J. D., Self-Assembling Multidomain Peptides: Design and Characterization of Neutral Peptide-Based Materials with $\mathrm{pH}$ and Ionic Strength Independent Self-Assembly. ACS Biomater. Sci. Eng. 2019, 5 (2), 977-985. DOI: 10.1021/acsbiomaterials.8b01348.

3. Lopez-Silva, T. L.; Leach, D. G.; Azares, A.; Li, I. C.; Woodside, D. G.; Hartgerink, J. D., Chemical functionality of multidomain peptide hydrogels governs early host immune response. Biomaterials 2020, 231, 119667. DOI: https://doi.org/10.1016/i.biomaterials.2019.119667.

4. Li, I. C.; Hartgerink, J. D., Covalent Capture of Aligned Self-Assembling Nanofibers. J. Am. Chem. Soc. 2017, 139 (23), 8044-8050. DOI: 10.1021/jacs.7b04655.

5. Judd, N. P.; Allen, C. T.; Winkler, A. E.; Uppaluri, R., Comparative Analysis of Tumor-Infiltrating Lymphocytes in a Syngeneic Mouse Model of Oral Cancer. Otolaryng. Head Neck 2012, 147 (3), 493-500. DOI: 10.1177/0194599812442037. 